\title{
Corynebacterium diphtheriae endocarditis
}

\author{
Fernando Peixoto Ferraz de Campos ${ }^{a}$, Vilma Takayasu ${ }^{a}$, Hugo Deutsch ${ }^{a}$, \\ Henrique Lane Staniaka ${ }^{a}$ Silvia Regina dos Santos ${ }^{b}$
}

Campos FPF, Takayasu V, Deutsch H, Staniak HL, Santos SR. Corynebacterium diphtheriae endocarditis. Autopsy Case Rep [Internet]. 2011;1(1):53-8.

\section{ABSTRACT}

The authors report the case of a 69-year-old man with an acute toxemic and febrile syndrome. Immediately after admission, the patient's mental status rapidly deteriorated. A faint mitral systolic murmur was detected and splenomegaly was observed on ultrasound examination. Laboratory screening revealed azotemia, AST and ALT elevation, leukocytosis, thrombocytopenia, elevated erythrocyte sedimentation rate as well as C-reactive-protein. Urinalysis showed proteinuria, leukocyturia and hematuria. A transesophageal echocardiogram showed large vegetations attached to both mitral leaflets with mild regurgitation. Blood culture yielded slow-growing Gram-positive bacillus which was later identified as a nontoxigenic Corynebacterium diphtheriae sorovar gravis. The authors emphasize the importance of identifying any bacteria isolated in blood samples particularly when an infective endocarditis diagnosis is suspected and call attention to the increasing number of reports related to this pathogen. Patient's clinical outcome was favorable after a long period of antibiotic therapy.

Keywords: Infectious endocarditis; Corynebacterium diphtheriae.

\section{INTRODUCTION}

Due to the diversity of clinical presentation as well as its high mortality rate, reaching up to $15 \%$ of the cases ${ }^{1}$, infectious endocarditis has always been a reason of concern.

When typical findings are present, it is not difficult to establish the diagnosis. However, some- times it is difficult to meet Dukes' diagnosis criteria $^{2}$, due to unfavorable conditions - nonspecific clinical presentation, impossibility to identify the etiological agent, limitations of the transthoracic echocardiography to show vegetations or perivalvular complications, unavailability of transesophageal echocardiography.

\footnotetext{
${ }^{a}$ Department of Internal Medicine - Hospital Universitário - Universidade de São Paulo, São Paulo/SP - Brazil.

b Clinical Pathology Laboratory - Hospital Universitário - Universidade de São Paulo, São Paulo/SP - Brazil.
} 
The microbiological diagnosis is fundamental for the specific treatment. When the blood culture shows the growth of Gram-positive bacillus, the clinician faces the dilemma of whether or not valuing this information, since this bacteriological group comprises the contaminants (usual components of normal human skin flora ${ }^{3,4}$ ), and not a microorganism typically related to infectious endocarditis. Furthermore, if the routine identification for Grampositive bacillus is unavailable, the microbiologist can erroneously consider it as a contaminant.

We report a case of native-valve infectious endocarditis, in an adult male patient, caused by a Gram-positive bacillus later identified as Corynebacterium diphtheriae.

\section{CASE REPORT}

A 69 year-old man, white, married, dog trainer, was admitted to the general ward of Hospital Universitário, with fever and mental confusion that started twenty four hours before the admision. The patient, previously healthy, reported a chest discomfort of the upper respiratory tract and cough for 20 days, which disappeared after three days of symptomatic treatment. On admission body temperature was $\left(39.8^{\circ} \mathrm{C}\right)$, arterial pressure of 90 / $60 \mathrm{mmHg}$, heart and pulse rate of 70 beats per minute, respiratory rate of 33 incursions per minute. The patient was toxemic, confused, temporally disoriented. Neck stiffness or any motor deficits were absent. Examination of the lungs revealed decreased breath sounds and rales were detected in the right lung base; the cardiovascular auscultation revealed a grade II/IV protomesosystolic murmur in the mitral area, without radiation. Petechiae were observed in the pretibial area over the shin.

After a set of blood culture was drawn, antibiotic therapy was initiated with ceftriaxone and clarithromycin based on the assumption of sepsis by a possible lung infection.

The laboratory exams follow-up is shown in Table 1. The bone marrow aspirate was found to be hypercellular mainly due to the granulocytic component. Serology for leptospirosis was negative and the serologies for hepatitis $B$ and $C$ showed: negative HbsAg; positive anti-HBs; positive anti-HBc (IgG) and negative anti-HCV. Results for anti-nuclear factor and rheumatoid factor were both negative. The urinary sediment revealed proteinuria, $147 \mathrm{mg} / \mathrm{dl}$; leukocytes, 13000/ $\mathrm{mm}^{3}$; erytrocytes, $72000 / \mathrm{mm}^{3}$ and presence of white cell casts. Slight increase of the cardiac silhouette and pulmonary congestion were observed on the chest X-ray, (Figure 1). Electrocardiogram was normal. A mild splenomegaly was shown by the abdominal ultrasound examination.

The transthoracic echocardiogram revealed normal functioning of the left ventricle with ejection fraction of $69 \%$, mitral valve thickening and prolapse with mild regurgitation. Due to the clinical suspicion of infectious endocarditis a transesophageal echocardiogram was performed revealing two vegetations attached to the mitral leaflets $(10 \times 5 \mathrm{~mm}$ and $11 \times 5 \mathrm{~mm})$ and significant mitral valve insufficiency (Figure 2).

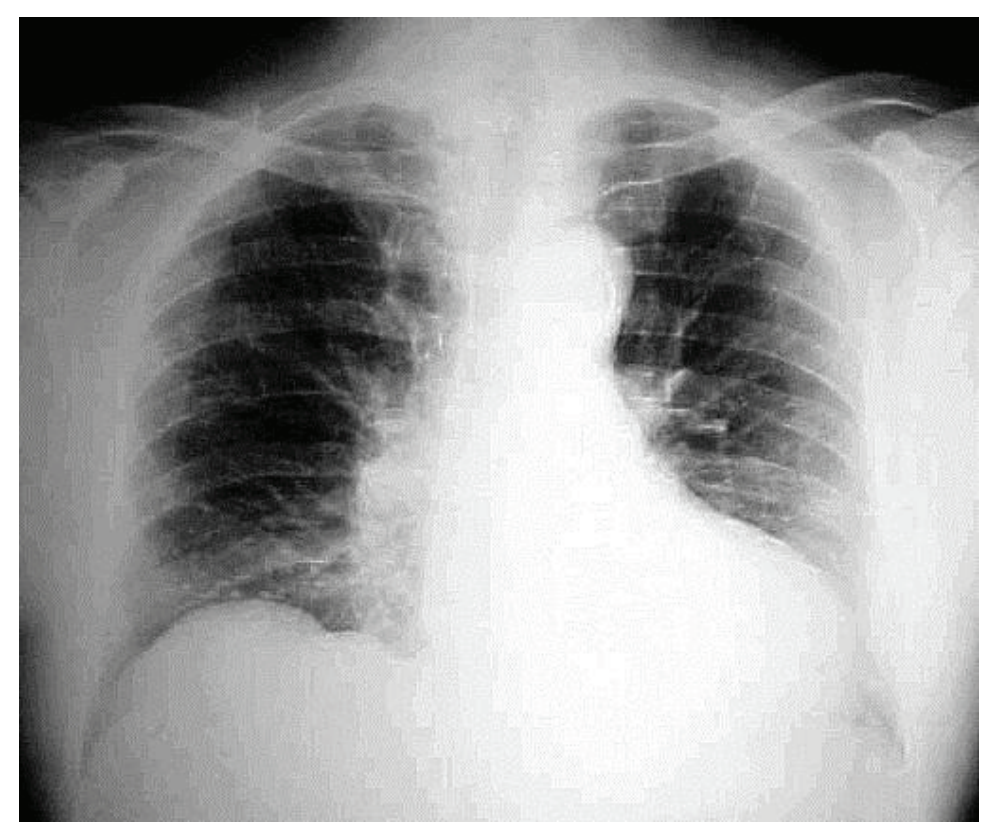

Figure 1 - Chest X-ray - showing increased cardiac silhouette and mild lung congestion. 


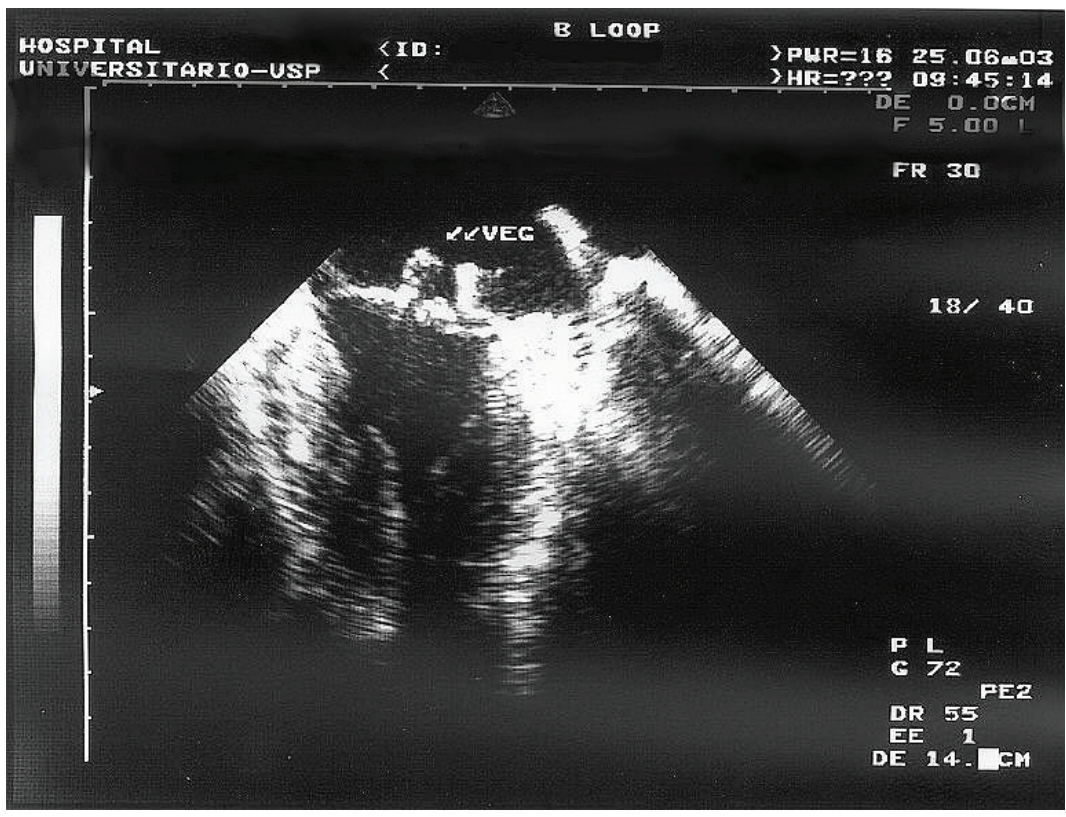

Figure 2 - Transesophageal Echocardiography showing images compatible with vegetations attached to both mitral valve leaflets.

The blood culture showed bacterial growth on the second day of incubation. However, due to the slow-growth of the bacterial colonies the identification was delayed. Microscopy of the isolate, by Gram stain method, showed coryneform Gram-positive bacilli. The catalase reaction was positive. Biochemical identification of the isolate was accomplished through the system API Coryne® (bioMérieux 69280 Marcy-l'Étoile/ France), showing pronounced negativity for the pyrazinamidase test, as well as the glucose, maltose and glycogen fermentation tests. The fermentation of the latter substrate differentiates the gravis biotype from the other biotypes.

Evaluation of diphtheric-toxin production was accomplished by the technique of Colman et $\mathrm{al}^{5}$, using the anti-diphtheria serum supplied by Dr. Hisako Gondo Higashi from Instituto Butantã (SP, Brazil), and yielded a negative result.

Genomic amplification reactions to detect tox $A$ and toxB genes, which codify sub-unities $A$ and $B$ of the diphtheria toxin respectively, were accomplished by the technique of Nakao et $\mathrm{al}^{6}$, yielding negative results. These microbiological results characterize Corynebacterium diphtheriae variety gravis, non-toxigenic.
The patient's clinical course was favorable. $\mathrm{He}$ was maintained on the same antimicrobial regimen as on admission, namely, ceftriaxone for 26 days and clarithromycin in the first 14 days. Due to recrudescence of fever (on the $22^{\text {nd }}$ day of hospitalization), even without deterioration of clinical and laboratorial conditions, nor evidence of any complication, the antibiotic regimen was modified to amikacin and crystalline penicillin, which were administered for 14 and 28 days, respectively. The patient's response to the new antibiotics was positive, since fever and other symptoms finally subsided. In addition, after five weeks of hospitalization, the transesophageal echocardiogram revealed a decrease in vegetations' size $(8 \times 5 \mathrm{~mm}$ and $9 \times 4 \mathrm{~mm})$ but persistence of significant mitral insufficiency. 
Table 1 - Laboratory tests follow-up.

\begin{tabular}{|c|c|c|c|c|c|c|c|c|}
\hline $\begin{array}{l}\text { Days of } \\
\text { hospitalization }\end{array}$ & $\mathbf{0}$ & 1 & 3 & 4 & 5 & 8 & 15 & 30 \\
\hline $\mathrm{Hb}(\mathrm{g} / \mathrm{dl}) / \mathrm{Ht}(\%)$ & $13.7 / 42$ & $11.5 / 35$ & $11 / 31$ & $11 / 33$ & $10.7 / 31$ & $10.9 / 32$ & $9.4 / 28$ & $9.3 / 27$ \\
\hline Platelets $/ \mathrm{mm}^{3}$ & 78000 & 42000 & 29000 & 39000 & 64000 & 225000 & 427000 & 291000 \\
\hline Leukocytes $/ \mathrm{mm}^{3}$ & 9000 & 9600 & 13400 & 15200 & 16000 & 24000 & 10000 & 8000 \\
\hline Myelocytes (\%) & - & - & - & 1 & 2 & - & - & - \\
\hline Metamyelocytes(\%) & - & - & - & 4 & 2 & - & - & - \\
\hline Band-neutrophils (\%) & 35 & 16 & 3 & 4 & 10 & 1 & - & 1 \\
\hline $\begin{array}{l}\text { Segmented-neutr. } \\
(\%)\end{array}$ & 56 & 76 & 76 & 58 & 59 & 85 & 68 & 68 \\
\hline Lymphocytes (\%) & 8 & 7 & 14 & 19 & 12 & 7 & 22 & 15 \\
\hline Urea (mg/dl) & 105 & 132 & 70 & 61 & 51 & 52 & 40 & 47 \\
\hline Creatinine (mg/dl) & 2.1 & 2.7 & 1.9 & 1.8 & 1.6 & 1.6 & 1.4 & 1.5 \\
\hline $\mathrm{AST}(\mathrm{u} / \mathrm{L})$ & & 406 & 71 & 105 & 93 & 67 & 55 & 48 \\
\hline ALT (mg/dl) & & 378 & 163 & 186 & 189 & 142 & 83 & 72 \\
\hline F Alkaline (u/L) & & 130 & 341 & & 742 & 717 & 509 & 323 \\
\hline Gamma GT (u/L) & 28 & & 150 & & 268 & 286 & 140 & 62 \\
\hline $\mathrm{DHL}(\mathrm{u} / \mathrm{L})$ & 2855 & 1928 & 823 & 844 & 788 & 696 & 437 & - \\
\hline CK (u/L) & & 517 & 152 & 55 & 37 & - & - & - \\
\hline
\end{tabular}

AST: aspartate aminotransferase; ALT: alanine aminotransferase; FA: alkaline phophatase; Gamma GT: gamma glutamil transferase; DHL: lactate dehydrogenase; CPK: creatine kinase; Hb: hemoglobin; Ht: hematocrit.

\section{DISCUSSION}

Diphtheria still remains an endemic disease in Brazil despite the reduction in the number of reported new cases. The vaccination coverage with the DPT vaccine raised from $66 \%$ in 1990 to more than $90 \%$ in $2002^{7}$.

Immunization with the diphtheria toxoid provides protection against the diphtheria toxin but does not prevent the carriage of the Corynebacterium diphtheriae in oro or nasopharynx ${ }^{8,9}$. People with poor hygiene standards, homeless, intravenous drug abusers, alcoholics and cirrhotic patients represent the major carriers of Corynebacterium diphtheriae. ${ }^{3,10,11,12,13}$
In a recent review of the English medical literature, 49 cases of infectious endocarditis by Corynebacterium diphtheriae, occurring from 1950 to 2000 , were described. ${ }^{13}$ Since 2000, 11 more cases (including the present report) were described, totalizing 60 cases. These cases reveal a predominant incidence among males (3:1), and younger individuals ( $81 \%$ of the cases developed in men under 40 years). The age distribution of infected individuals was from 4 to 69 years, and the mean age was 24 years $^{14}$.

Endocarditis by Corynebacterium diphtheriae usually occurs in previously healthy individ- 
uals; $42 \%$ of the patients did not have any valvular disease or other risk factors. ${ }^{13}$ The incidence is higher on left heart valves, with preference for the mitral valve. Frequently, the vegetations are large, facilitating septic embolization and the occurence of aneurysms. ${ }^{15,16}$ This fact probably explains the high morbimortality rates: up to $58 \%$, for the occurrence of complications, and $37 \%$, for fatal outcome. Depending on the biotype involved, these rates can vary accordingly: endocarditis by Corynebacterium diphtheriae biovar gravis was fatal in $19 \%$ of the cases, but when infection was caused by Corynebacterium diphtheriae biovar mitis, the mortality rate reached $64 \%$ of cases. ${ }^{13}$ Untill 1950 almost all invasive strains produced toxins. However, after 1950 only seven of 52 invasive cases were caused by toxin-producing Corynebacterium diphtheriae: three not biotyped; two were identified as biovar gravis; one, as biovar mitis and the last one, as biovar intermedius. ${ }^{13,17,18}$

The isolation of Corynebacterium sp in blood cultures is usually interpreted as contamination ${ }^{3,13}$, and such a procedure can hinder the endocarditis diagnosis, especially in patients not showing the typical clinical picture, contributing to the morbimortality of this disease.

The current case report calls attention to its acute presentation form, with signs of significant toxemia, as well as laboratory alterations compatible with a septicemic picture: thrombocytopenia, increase of aminotransferases, and renal function impairment with possible glomerulitis. Renal function impairment probably ocurred due to a combination of factors, such as sepsis, persistent antigenemia, hypotension and hypovolemia. The transthoracic echocardiogram was not sensitive (sensitivity $60-70 \%$ and specificity $98 \%$ ) for the diagnosis of infectious endocarditis, thus pointing to the need of a transesophageal examination (sensitivity $75-95 \%$ and specificity $85-98 \%) .^{2}$ The transesophageal echocardiogram showed that large vegetations were already present by the onset of the disease. Despite a favorable clinical course, we observed that the residual valvular lesion remained functionally important, as the echocardiographic control revealed that the mitral insuffiency, once mild, became significant by the time of the patient's hospital discharge. Regarding this lesion, it is important to observe that the patient did not show any signs of congestive heart failure during the entire hospital stay.
It is important to emphasize that, whenever an infectious endocarditis diagnosis is possible, the identification of any agent isolated in blood culture is fundamental. The bacteria identified as a Gram-positive bacillus could be precociously misinterpreted as contaminant. In this report we showed that the isolated bacterium was not a contaminant, later identified as Corynebacterium diphtheriae. Even if it were a non-diphtheric Corynebacterium, one should not disregard it as a possible endocarditis causative agent. These microorganisms are recognized as causative of community-acquired $^{4}$ or hospital-acquired ${ }^{19}$ infectious endocarditis, in patients with previous structural heart disease or prosthetic valves, intravenous catheter users, as well as the immunodeficient patients. ${ }^{10,12}$ In 1995, a review reported 126 cases of infectious endocarditis caused by a non-diphtheric Corynebacterium. Of these cases, $58 \%$ occurred in native-valves and $42 \%$ in prosthetic valves, and the main causative agents were Corynebacterium pseudodiphtericum and Corynebacterium jeikeium. ${ }^{10}$

In cases of infection by non-diphtheric Corynebacterium, the etiological identification is frequently impaired by the slow bacterial growth. Sometimes, an incubation period of 7 to 15 days ${ }^{12,20}$ is needed to detect this growth, and some cases demand specific lipophilic culture medium which is not routinely available. ${ }^{20,21}$ Additionally, nondiphtheric Corynebacteria are major components of human cutaneous and mucosal flora $3,12,17,19$ and as such, disregarded as contaminants. ${ }^{12,17}$

Corynebacteria are considered to be responsible for 0.2 to $0.4 \%$ of infectious endocarditis cases in native valve, $9 \%$ of cases in early prosthetic valves and $4 \%$ in late prostheses. ${ }^{22}$ , 23 The observed increase in case reports of infectious endocarditis by Corynebacterium $s p$ is related to a combination of factors: better taxonomic knowledge of these agents, improvement of identification techniques, increasing awareness among clinicians and microbiologists to the importance of these agents, and finally, the increasing number of immunocompromised patients. $^{21}$

\section{ACKNOWLEDGEMENTS}

The authors are grateful to Dr. Jorge Luiz Mello Sampaio - Laboratório Fleury, São Paulo SP, Brazil - Fleury for the microbiological studies. 


\section{REFERÊNCIAS}

1. Young SEJ. Aetiology and epidemiology of infective endocarditis in England and Wales. J Anitimicrob Chemother. 1987; 20(suppl A):7-14.

2. Mylonakis E, Calderwood S B. Infective endocarditis in adults. N Engl J Med. 2001;345(18):1318-28.

3. Rivero M, Quereda C, Martin-Dávila P, Moya J L, Navas E, Moreno S. [Corynebacterium pseudodiphtericum endocarditis]. Enferm Infecc Microbiol Clin. 2003;21(2):11620. Spanish.

4. Pessanha B, Farb A, Lwin T, Lloyd B, Virmani R. Infectious endocarditis due to Corynebacterium xerosis. Cardiovasc Pathol. 2003;12(2):98-101.

5. Colman G, Weaver E, Efstratiou A. Screening tests for pathogenic corynebacteria. J Clin Pathol. 1992;45(1):46-8.

6. Nakao H, Popovic T. Development of a direct PCR assay for detection of the diphtheria toxin gene. J Clin Microbiol. 1997;35(7):1651-5.

7. Ministério da Saúde (BR). Secretaria de Vigilância em Saúde. Saúde Brasil 2004: uma análise da situação de saúde. Brasília, DF; 2004 [cited 2010 Dec 16]. Chapter 6: Situação da prevenção e controle das doenças transmissíveis no Brasil. Available from: http://portal.saude.gov.br/ portal/arquivos/pdf/capitulo6_sb.pdf

8. Afghani B, Stutman HR. Bacterial arthritis caused by Corynebacterium diphtheriae. Pediatr Infect Dispneia J. 1993;12(10):881-2.

9. Edward DG, Allison VD. Diphtheria in the immunized with observations on a diphtheria-like disease associated with non-toxigenic strains of Corynebacterium diphtheriae. J Hyg. 1951;49(2/3):205-19.

10. Petit PLC, Bok JW, Thompson J, Buiting AG, Coyle MB. Native-valve endocarditis dur to CDC coryneform group ANF-3: report of a cse and review of corynebacterial endocarditis. Clin Infect Dis.1994;19(5):897-901.

11. Funke G, Altwegg M, Frommelt L, von GraevenitzA. Emergence of related nontoxigenic Corynebacterium diphtheriae biotype mitis strains in Western Europe. Emerg Infect Dis. 1999;5(3):477-80.

12. Van Scoy RE, Cohen SN, Geraci JE, Wasington JA 2nd. Coryneform bacterial endocarditis: difficulties in diagnosing and treatment, presentation of three cases, and re- view of literature. Mayo Clin Proc. 1977;52(4):216-9.

13. Belko J, Wessel DL, Malley R. Endocarditis caused by Corynebacterium diphtheriae: case report and review of the literature. Pediatr Infect Dis J. 2000;19(2):159-63.

14. Schnell D, Beyler C, Lanternier F, Lucron H, Lebeaux D, et al. Nontoxigenic Corynebacterium diphteriae as a rare cause of native endocarditis in childhood. Ped Infec Dis J. 2010;29(9):886-8.

15. Holthouse DJ, Power B, Kermode A, Golledge C. Nontoxigenic Corynebacterium diphtheriae:two cases and review of the literature. J Infect. 1998;37(1):62-6.

16. Tyley SM, Kociuba KR, Heron LG, Munro R. Infective endocarditis due to Nontoxigenic Corynebacterium diphtheriae: report of seven cases and review. Clin Infect Dis. 1993;16(2):271-5.

17. Durandy $Y$, Hulin S. [Acute Corynebacterium diphtheriae aortic endocarditis with negative blood culture]. Arch Pediatr. 1999:6(11):1196-8. French.

18. Benes J, Picha D, Kabelkova M, Dzupova O, Horova B, Gabrielova A. Infective endocarditis caused by unusual gram-positive pathogens. Folia Microbiol. 2002;47(6):73741.

19. Knox KL, Holmes A. Nosocomial endocarditis caused by Corynebacterium amycolatum and other Nondiphtheriae Corynebacteria. Emerg Infect Dis. 2002;8(1):97-9.

20. Sewell DL, Coyle MB, Funke G. Prosthetic valve endocarditis caused by Corynebacterium afermentans subsp. lipophilum (CDC coryneform group ANF-1). J Clin Microbiol. 1995;33(3):759-61.

21. Clayes G, Vanhouteghen H, Riegel P, Wauters G, Hamerlynck R, Dierick J, et al. Endocarditis of native aortic and mitral valve de to Corynebacterium accolens: report of a case and application of phenotype and genotypic techniques for identification. J Clin Microbol. 1996; 34(5):1290-2.

22. Wilson APR. The return of Corynebacterium diphtheriae: the rise of nontoxigenic strains. J Hosp Infect. 1995;30(suppl):306-12.

23. Berbari EF, Cockerill FR 3rd, Steckelberg JM. Infective endocarditis due to unusual or fastidious microorganisms. Mayo Clin Proc 1997;72(6):532-42.

\section{Conflict of interest: None}

Submitted on: 16 th de December 2010

Accept on: 24 deth February 2011

Correspondence: Divisão de Clínica Médica

Av. Prof. Lineu Prestes, 2565 - Cidade Universitária - São Paulo - SP - Brazil

CEP: 05508-000 - Phone: +55.011. 3091.9200

E-mail: ffcampos@usp.br 